\title{
REPRESENTACIONES EXTREMALES
}

\section{Tomás Núñez Lay ${ }^{9}$ \& Helmuth Villavicencio Fernández ${ }^{10}$}

Resumen: En el presente trabajo se muestra la relación de un conjunto convexo con sus elementos extremos, lo que se conoce como representaciones extremales. Extendemos el teorema de Krein-Milman en dimensión finita, para un convexo cualquiera.

Palabras clave: Caracterización extremal. Extensiones del teorema de KreinMilman. Coterminalidad. Análisis convexo.

\section{REPRESENTATIONS EXTREMALS}

\begin{abstract}
In this work, we show the relation about a convex set with their extremes elements. We extended the Kein-Milman theorem in finite dimension for arbitrary convex set.
\end{abstract}

Key words: Extremal characterization. Extensions of Krein-Milman theorem. Coterminalidad. Convex analysis.

\section{Introducción}

Dado un conjunto convexo $C \subseteq \mathbb{R}^{n}$. Si denotamos por $C o(C)$ al conjunto de sus combinaciones convexas, llamado casco convexo de $C$, se tiene que $C=C o(C)$. Es decir, podemos obtener el conjunto $C$ a partir de las combinaciones convexas de sus elementos. Luego surge la siguiente pregunta ¿podremos expresar $C=C o(S)$ para algún subconjunto propio $S$ de $C$ ? la cual, de ser posible responderla, origina de modo natural la siguiente ¿podríamos tomar $S$ mínimo? donde la minimalidad se entiende en el sentido de inclusión: si existe $B \subseteq C$ tal que $C=C o(B)$ entonces $S \subseteq B$.

Un subconjunto convexo $F$ de $C$ se dice extremal si $C \backslash F$ es aún convexo. Cuando $F$ es unitario, se llama punto extremo y si $F$ es un rayo se dice rayo extremo. En principio, un candidato para $S$ sería la frontera de $C$, pero como ocurre en el caso compacto los puntos extremos son suficientes.

Teorema 1.1 (Krein-Milman) Todo conjunto no vacio, convexo y compacto de $\mathbb{R}^{n}$, es el casco convexo de sus puntos extremos.

Prueba. Ver [4].

Luego notamos que para poder responder las interrogantes planteadas en el caso no compacto podemos considerar $S$ en función de los elementos extremales de $C$ (puntos extremos y rayos extremos). Dichas expresiones de $S$ generan para $C=C o(S)$ lo que denominaremos: representaciones extremales. Dado que los elementos extremales de $C$ están incluídos en la frontéra de $C$, entonces no podremos obtener representaciones extremales para conjuntos abiertos, hecho por el cual estudiaremos primero el caso en que $C$ es cerrado y luego lo debilitaremos. Notemos que cada representación extremal generaliza, en cierto modo, al

\footnotetext{
${ }^{9}$ UNMSM, Facultad de Ciencias Matemáticas, e-mail: tnunezl@gmail.com

${ }^{10}$ UNMSM, Facultad de Ciencias Matemáticas, e-mail: helmuth.villavicencio@gmail.com
} 
teorema de Krein-Milman, más aún obtendremos una prueba de éste como caso particular.

Sea $C \subseteq \mathbb{R}^{n}$ no vacío y convexo, denotamos por $\Lambda[C]$ a la menor variedad lineal que contiene a $C$. La dimensión de $C, \operatorname{dim}(C)$, será la dimensión de $\Lambda[C]$. El interior relativo de $C$, denotado por $r i(C)$ es el interior de $C$ relativo a $\Lambda[C]$. La frontera relativa de $C$, denotada por $\operatorname{cl}(C)$ se define como $\operatorname{cl}(C)=\bar{C} \backslash r i(C)$. El cono asintótico de $C$, denotado por $C_{\infty}(C)$ es el conjunto de las direcciones que constituyen $C$. Dado $x \in C$ definimos $C_{\infty}(x)=\left\{u \in \mathbb{R}^{n}: x+\lambda u \in C, \forall \lambda \geq 0\right\}$. El espacio de linealidad $L$, de $C$ es definido por $L=C_{\infty}(C) \cap-C_{\infty}(C)$. El lector interesado en mayores detalles puede consultar las referencias [1], [2], [3] y [7].

\section{Representaciones extremales}

Daremos ahora las fórmulas de descomposición extremal de un conjunto convexo. Empezaremos dando un criterio para obtener $C=\operatorname{Co}(\operatorname{cl}(C))$.

Proposición 2.1 Sea $V \subseteq \mathbb{R}^{n}$ no vacío y convexo. Si $V=\operatorname{cl}(B)$ donde $B$ es un conjunto convexo tal que $\operatorname{int}(B) \neq \emptyset$, entonces $V$ es un hiperplano en $\mathbb{R}^{n}$ y $B \cup V$ es un semi-espacio cerrado de $\mathbb{R}^{n}$.

Prueba. Ver [5].

Definición 2.2 (Segmento maximal) $S e a C \subseteq \mathbb{R}^{n}$ convexo y no vacío. Dados $a, b \in C$ tales que $\mu=a-b \neq 0$ se dice que $[a, b]$ es un segmento maximal de $C$ en la dirección $\mu$, si el conjunto $S=\{\lambda \in \mathbb{R}: a+\lambda \mu \in C\}$ es acotado y además $\operatorname{Sup}(S)=1$ e $\operatorname{Inf}(S)=0$.

Proposición 2.3 Sea $C \subseteq \mathbb{R}^{n}$ no vacio, convexo y cerrado. Entonces son equivalentes:

i. existe en $C$ un segmento maximal.

ii. $C$ es la unión de sus segmentos maximales.

iii. $C \neq \Lambda[C]$ y $C$ no es un semi-espacio en $\Lambda[C]$.

Prueba. ( $i$ ) implica (ii) Por la existencia del segmento maximal, existen $w \in C$ y $\mu \neq 0$ tales que $A_{w}=\left\{\lambda \in \mathbb{R}^{+}: w+\lambda \mu \in C\right\}$ es acotado. Sea $z \in C$, si el conjunto $A_{z}=\left\{\lambda \in \mathbb{R}^{+}\right.$: $z+\lambda \mu \in C\}$ no es acotado, existe un rayo asintótico en $C$, entonces $\mu \in C_{\infty}(C)=C_{\infty}(w)$ luego $A_{w}$ no es acotado, lo cual es absurdo. Sean $\alpha_{1}=\operatorname{Inf} A_{z}, \alpha_{2}=\operatorname{Sup} A_{z}$ como $C$ es cerrado, $x=z+\alpha_{1} \mu, \quad y=z+\alpha_{2} \mu \in C$ y $A_{z} \subseteq[x, y]$, donde $x, y \in \operatorname{cl}(C)$ entonces

$$
C=\bigcup_{x, y \in c l(C)}[x, y]
$$

(ii) implica (iii) Por la existencia de segmentos maximales, no hay líneas en dichas direcciones luego $C \neq \Lambda[C]$ entonces $C$ no es un semi-espacio en $\Lambda[C]$.

(iii) implica $(i)$ Sea $K=\operatorname{cl}(C)$ y dado que $C$ no es un semi-espacio en $\Lambda[C]$ entonces se tiene que $K$ no es convexo.

Entonces existen $p, q \in K$ tales que $[p, q] \nsubseteq K$ por lo tanto existe $x \in[p, q] \backslash K \subseteq C \backslash K$, luego $x \in \operatorname{ri}(C)$. Supongamos que $[p, q]$ no es un segmento maximal en $C$, luego si $\Psi=\Lambda([p, q])$ entonces existe $w \in(\Psi \backslash[p, q]) \cap C$ y para algún punto, digamos $p$, se tiene $p \in[x, w)$ entonces $[x, w) \subseteq \operatorname{ri}(C)$ y por tanto $p \in \operatorname{ri}(C)$ luego $p \notin K$, absurdo. 
Lema 2.4 Sea $C \subseteq \mathbb{R}^{n}$ no vacío, cerrado, convexo no conteniendo líneas y tal que $\operatorname{dim}(C) \geq 2$, entonces $C=\operatorname{Co}(c l(C))$.

Prueba. Al no contener líneas, $C \neq \Lambda[C]$ y $C$ no es un semi-espacio en $\Lambda[C]$ pues $\operatorname{dim}(C) \geq 2$ y estamos en la condición (iii) de la proposición anterior entonces por (ii), $C$ es la unión de sus segmentos maximales de donde se sigue el resultado.

Teorema 2.5 Sea $C \subseteq \mathbb{R}^{n}$ no vacío, cerrado, convexo y no contiene líneas. Si $R x t(C)$ es la unión de los rayos extremos de $C$, entonces

$$
C=\operatorname{Co}(\operatorname{Ext}(C) \cup \operatorname{Rxt}(C))
$$

Prueba. Basta probar que $C \subseteq C o(\operatorname{Ext}(C) \cup \operatorname{Rxt}(C))$. Haremos la prueba por inducción sobre la dimensión $n$ de $\Lambda[C]$. Para $n \leq 1$ la afirmación es trivialmente cierta. Supongamos que la afirmación fue probada para $n=k$ y consideremos que $\Lambda[C]$ tiene dimensión $(k+1)$. Por el lema anterior, $C=\operatorname{Co}(\operatorname{cl}(C))$. Sea $x \in \operatorname{cl}(C)$ y sea $H$ un hiperplano en $\Lambda[C]$ de soporte para $C$ en $x$, entonces por la hipótesis inductiva para $H \cap C$ se sigue que

$$
x \in H \cap C=C o(\operatorname{Ext}(H \cap C) \cup \operatorname{Rxt}(H \cap C) \subseteq \operatorname{Co}(\operatorname{Ext}(C) \cup \operatorname{Rxt}(C))
$$

Entonces $x \in \operatorname{Co}(\operatorname{Ext}(C) \cup \operatorname{Rxt}(C)$ para todo $x \in \operatorname{cl}(C)$ por lo tanto

$$
C=C o(c l(C)) \subseteq C o(\operatorname{Ext}(C) \cup \operatorname{Rxt}(C)) .
$$

Corolario 2.6 Sea $C \subseteq \mathbb{R}^{n}$ no vacio, cerrado, convexo y tal que no contiene líneas. Se tiene

$$
C=C o(\operatorname{Ext}(C))+C_{\infty}(C)
$$

Prueba. Dado $x_{0} \in \operatorname{Rxt}(C)$, existe un rayo extremo $\rho$ de $C$ que contiene a $x_{0}$ y es de la forma $\rho=\{z+\lambda y \in C: \lambda \geq 0\}$ donde $z \in \operatorname{Ext}(C)$ e $y \neq 0$. Luego $y \in C_{\infty}(z)$, como $C$ es cerrado se tiene $y \in C_{\infty}(C)$ y por ser este último conjunto un cono, entonces $x_{0} \in \rho \subseteq z+C_{\infty}(C)$ de donde $\operatorname{Rxt}(C) \subseteq \operatorname{Ext}(C)+C_{\infty}(C) \subseteq C$, luego $\operatorname{Ext}(C) \cup \operatorname{Rxt}(C) \subseteq \operatorname{Ext}(C)+C_{\infty}(C) \subseteq C$ y usando lo obtenido en el teorema anterior

$$
C=\operatorname{Co}(\operatorname{Ext}(C) \cup \operatorname{Rxt}(C)) \subseteq \operatorname{Co}\left(\operatorname{Ext}(C)+C_{\infty}(C)\right) \subseteq C
$$

Dado que $C_{\infty}(C)$ es convexo, se tiene $C=C o(\operatorname{Ext}(C))+C_{\infty}(C)$.

Corolario 2.7 Sea $C \subseteq \mathbb{R}^{n}$ no vacío, cerrado, convexo y tal que no contiene líneas. Entonces, $C=C o(\operatorname{Ext}(C))$ si y sólo si $\operatorname{Rxt}(C)=\emptyset$.

Prueba. Basta notar que $C_{\infty}(C)=\{0\}$ si y sólo si $\operatorname{Rxt}(C)=\emptyset$.

Para el caso que el conjunto contenga líneas, notemos que dado un punto en la línea existen dos direcciones opuestas, luego $C_{\infty} \cap-C_{\infty} \neq\{0\}$, es decir el espacio de linealidad $L$ no es trivial. Esto nos lleva a la siguiente fórmula de representación extrema.

Teorema 2.8 Sea $C \subseteq \mathbb{R}^{n}$ no vacío, cerrado y convexo. Si $L$ es el espacio de linealidad de $C$ y $C_{L}=C \cap L^{\perp}$, entonces

$$
C=L+C o\left(\operatorname{Ext}\left(C_{L}\right)\right)+C_{\infty}\left(C_{L}\right)
$$


Prueba. Como $L$ es el espacio de linealidad de $C$ se verifica $C=L+C \cap L^{\perp}$, luego basta mostrar que $C_{L}=C \cap L^{\perp}=\operatorname{Co}\left(\operatorname{Ext}\left(C_{L}\right)\right)+C_{\infty}\left(C_{L}\right)$ y por el corolario ?? es suficiente probar que $C \cap L^{\perp}$ no contiene líneas.

Afirmación. $C_{L}=C \cap L^{\perp}$ no contiene líneas.

En efecto; supongamos exista una recta $\Gamma \subseteq C_{L}$ luego existe $x_{0} \neq 0$ con $x_{0} \in C_{\infty}\left(C_{L}\right)$ y dado $z \in \Gamma$, como $C$ es cerrado entonces $x_{0} \in C_{\infty}(z)$ y al ser $\Gamma$ una recta $-x_{0} \in C_{\infty}(z)$, entonces $x_{0} \in L$. Luego se tiene $z+x_{0} \in L^{\perp}$ (pues $x_{0} \in C_{\infty}(z)$ ), $z \in L^{\perp}$ (pues $z \in \Gamma$ ) y $0 \neq x_{0} \in L$. Entonces

$0=\left\langle z+x_{0}, x_{0}\right\rangle=\left\langle z, x_{0}\right\rangle+\left\langle x_{0}, x_{0}\right\rangle=\left\|x_{0}\right\|^{2}$ de donde $x_{0}=0$, absurdo.

Veamos ahora que en efecto el teorema ?? es una extensión del teorema de KreinMilman. Sea $C \subseteq \mathbb{R}^{n}$ no vacío, compacto y convexo. Como $C$ es acotado, $C_{\infty}(C)=\{0\}$ entonces $L=\{0\}$. Por el teorema anterior se tiene $C=C o\left(\operatorname{Ext}\left(C_{L}\right)\right)$, donde $C_{L}=C \cap L^{\perp}$, por lo tanto

$$
C=C o\left(\operatorname{Ext}\left(C_{L}\right)\right)=\operatorname{Co}\left(\operatorname{Ext}\left(C \cap L^{\perp}\right)\right)=\operatorname{Co}\left(\operatorname{Ext}\left(C \cap \mathbb{R}^{n}\right)\right)=\operatorname{Co}(\operatorname{Ext}(C)) .
$$

Por otro lado, notemos que si $C$ contiene un rayo extremo $\rho$, entonces la representación $C=C o(\operatorname{Ext}(C) \cup R x t(C))$ no satisface la noción de minimalidad puesto que es suficiente considerar cualquier parte no acotada del rayo $\rho$. Esto motiva a la siguiente definición.

Definición 2.9 (Coterminalidad) Sea $C \subseteq \mathbb{R}^{n}$ no vacio y convexo. Sea $\rho=\left\{\rho_{r} \in C: \rho_{r}=\right.$ $z+r w, r \geq 0\}$ un rayo extremo de $C$, donde $z \in \operatorname{Ext}(C)$ y $w$ es una dirección no nula. Decimos que $X \subseteq \bar{C}$ es coterminal con el rayo extremo $\rho$ de $C$, si $\operatorname{Sup}\left\{r: \rho_{r} \in X\right\}=+\infty$.

La noción de coterminalidad permite caracterizar a los subconjuntos $X \subseteq C$ cuyas combinaciones convexas generan $C$, es decir $C o(X)=C$.

Teorema 2.10 Sea $C \subseteq \mathbb{R}^{n}$ no vacío, cerrado, convexo y no contiene líneas. Sea $X \subseteq C$ entonces $C o(X)=C$ si y sólo si $\operatorname{Ext}(C) \subseteq X$ y $X$ es coterminal con cada rayo extremo de $C$.

Prueba. Supongamos $C o(X)=C$ y sea $x \in \operatorname{Ext}(C) \subseteq C$ entonces $x \in C o(X)$ luego existen $x_{1}, \ldots, x_{k} \in X$ tales que

$$
x \in \operatorname{Co}\left\{x_{1}, \ldots, x_{k}\right\}=\operatorname{Co}\left\{x_{1}, \operatorname{Co}\left\{x_{2}, \ldots, x_{k}\right\}\right\}
$$

Entonces $x \in\left[x_{1}, y\right]$ donde $y \in C o\left\{x_{2}, \ldots, x_{k}\right\}$, y por ser $x$ punto extremo se tiene que $x=y=x_{1} \in X$ de donde $\operatorname{Ext}(C) \subseteq X$.

Consideremos el rayo extremo $\rho=\left\{\rho_{r}: r \geq 0\right\}$ de $C$, donde $\rho_{r}=z+r w, z \in \operatorname{Ext}(C)$ y $w$ es una dirección no nula. Dado que $\operatorname{Rxt}(C) \subseteq C$ resulta $\operatorname{Rxt}(C) \subseteq C o(X)$ entonces $\rho \subseteq C o(X)$. Si $\operatorname{Sup}\left\{r: \rho_{r} \in X\right\}<\infty$, llamando a este supremo $M$, basta tomar $r_{0}=M+1>0$, para que $\rho_{r_{0}} \notin X$ pero como $\rho_{r_{0}} \subseteq C o(X)$ existen $x_{1}, \ldots, x_{k} \in X$ tales que

$$
\rho_{r_{0}} \in \operatorname{Co}\left\{x_{1}, \ldots, x_{k}\right\}=\operatorname{Co}\left\{x_{1}, \operatorname{Co}\left\{x_{2}, \ldots, x_{k}\right\}\right\}
$$

Entonces $\rho_{r_{0}} \in\left[x_{1}, y\right]$ donde $y \in C o\left\{x_{2}, \ldots, x_{k}\right\}$, luego $\rho \cap\left[x_{1}, y\right] \neq \emptyset$ y por ser $\rho$ rayo extremo existen $r_{1}, r_{2}$ tales que $x_{1}=\rho_{r_{1}}, y=\rho_{r_{2}}$ entonces $r_{0} \leq r_{3}=\operatorname{má} x\left\{r_{1}, r_{2}\right\}$ luego $\rho_{r_{3}} \in \rho$ de donde $\rho_{r_{0}} \in X$, absurdo.

Recíprocamente, supongamos que $\operatorname{Ext}(C) \subseteq X$ y $X$ es coterminal con cada rayo extremo de $C$ entonces $\operatorname{Ext}(C) \subseteq \operatorname{Co}(X)$, veamos que $\operatorname{Rxt}(C) \subseteq \operatorname{Co}(X)$. Si existe $\rho \subseteq R x t(C)$ tal que $\rho \nsubseteq C \operatorname{Co}(X)$, donde $\rho=\{z+r w: r \geq 0\}$ y $z \in \operatorname{Ext}(C)$, entonces existe $r_{0} \geq 0$ tal que $\rho_{r_{0}}=z+r_{0} w \notin C o(X)$. Si existe $r_{1}>r_{0}$ tal que $\rho_{r_{1}} \in C o(X)$ dado que 
$z \in C o(X)$ entonces $\rho_{r_{0}} \in\left[z, \rho_{r_{1}}\right] \subseteq C o(X)$, absurdo. Por lo tanto para todo $r \geq r_{0}$ se cumple que $\rho_{r} \notin C o(X)$ entonces $\operatorname{Sup}\left\{r: \rho_{r} \in X\right\}<r_{0}<\infty$ lo cual contradice la hipótesis. Entonces $\operatorname{Ext}(C) \cup \operatorname{Rxt}(C) \subseteq C o(X)$ y por fórmula de representación extremal $C=C o(\operatorname{Ext}(C) \cup R x t(C)) \subseteq C o(X)$ por tanto $C=C o(X)$.

El teorema anterior muestra que si $\operatorname{Rxt}(C)=\emptyset$, entonces $\operatorname{Ext}(C)$ es el conjunto más pequeño de $C$ tal que $C=C o(\operatorname{Ext}(C))$.

\section{Krein-Milman fortalecido}

En la sección anterior, los teoremas 2.5 y 2.8 extienden débilmente al teorema de Krein-Milman, ya que en ellos se usa la hipótesis de cerradura para el conjunto convexo. Surge entonces la interrogante de poder extender el teorema cuando el conjunto convexo de $\mathbb{R}^{n}$, no sea cerrado. Consideremos el conjunto:

$$
B=\left\{(x, y, z) \in \mathbb{R}^{3}: x^{2}+y^{2}+z^{2} \leq 1, z>0\right\}
$$

claramente $B$ es convexo y no es cerrado. Notemos que todo punto $(x, y, z)$ de la esfera de radio unidad $S^{2}$, con $z>0$ es punto extremo de $B$, es decir $\operatorname{Ext}(B)=S^{2} \cap B$. Además se prueba que $B=C o(\operatorname{Ext}(B))$, a pesar de que $B$ no es cerrado. Es decir se satisface la conclusión del teorema de Krein-Milman. Pero, ¿qué tiene de particular este conjunto para poder obtener la conclusión del teorema de Krein-Milman? La respuesta la encontramos en las caras de $B$. Podemos notar que las caras de $B$ son de dimensión cero, es decir puntos. Así, toda cara de $B$ es un punto extremo de $B$. Sea $F$ una cara de $B$, entonces $F=\{b\}$ para algún $b \in \operatorname{Ext}(B)$ luego se tiene que $\operatorname{Ext}(F)$ es denso en $\operatorname{Ext}(\bar{F})$. Surge entonces una nueva interrogante: La condición que para cada cara $F$ de $B, \operatorname{Ext}(F)$ es denso en $\operatorname{Ext}(\bar{F})$ ¿será suficiente para decir que $B=C o(\operatorname{Ext}(B))$ ? La respuesta a esta interrogante es afirmativa en el caso acotado, más aún probaremos que para el caso no acotado, basta que se incluyan los rayos extremos de las caras.

Lema 3.1 Sea $A \subseteq \mathbb{R}^{n}$ convexo y compacto tal que $\operatorname{dim}(A)=p$. Todo punto del interior relativo de A pertenece al interior relativo del casco convexo de a lo más $2 p$ puntos extremos de $A$.

Prueba. Por el teorema de Krein-Milman se tiene $A=\operatorname{Co}(\operatorname{Ext}(A))$. Sea $x \in \operatorname{ri}(A) \sin$ pérdida de generalidad supongamos que $x=0$ (basta hacer una traslación). Por tanto $0 \in \operatorname{ri}(C o(\operatorname{Ext}(A)))$, sea $V$ el conjunto de las combinaciones lineales de a lo más $p-1$ puntos de $\operatorname{Ext}(A)$, luego $V \neq \Lambda[C]$. De donde existe una recta $L$ tal que $L \cap V=\{0\}$ y sean $w_{1}, w_{2}$ los puntos de $L$ que son frontera con $C o(\operatorname{Ext}(A))$. Para cada $i \in\{1,2\}$ sea $H_{i}$ el hiperplano de soporte de $\operatorname{Co}(\operatorname{Ext}(A))$ en $w_{i}$. Claramente $0 \in\left(w_{1}, w_{2}\right)$ y $w_{i} \in \operatorname{Co}\left(\operatorname{Ext}(A) \cap H_{i}\right)$, luego por teorema de Carathedory $w_{i}$ puede ser escrito como combinación convexa de a lo más $p$ puntos $v_{1}^{i}, \ldots, v_{p}^{i}$ de $\operatorname{Ext}(A) \cap H_{i}$ luego $w_{i}$ pertenece al interior relativo de $\operatorname{Co}\left(\left\{v_{1}^{i}, \ldots, v_{p}^{i}\right\}\right)$, pues $w_{i} \notin V$ donde $i \in\{1,2\}$. Entonces $x=0 \in \operatorname{ri}\left(\operatorname{Co}\left(\left\{v_{1}^{1}, \ldots, v_{n}^{1}, v_{1}^{2}, \ldots, v_{p}^{2}\right\}\right)\right)$.

Estamos en condiciones de enunciar y probar el resultado principal de esta sección, que caracteriza a los conjuntos convexos que satisfacen la tesis del teorema de Krein-Milman.

Teorema 3.2 Sea $A \subseteq \mathbb{R}^{n}$ no vacío, convexo y acotado. Entonces $A=C o(\operatorname{Ext}(A))$ si y sólo si, $\operatorname{Ext}(F)$ es denso en $\operatorname{Ext}(\bar{F})$, para cada cara $F$ de $A$.

Prueba. Primero, supongamos que $\operatorname{Ext}(F)$ es denso en $\operatorname{Ext}(\bar{F})$, para cada cara $F$ de $A$. Probaremos que $A=\operatorname{Co}(\operatorname{Ext}(A))$ y para esto usaremos inducción en la dimensión $d$ de $A$. Para $d \leq 1$ es trivial pues $A$ sería un punto o un segmento de recta cerrado. Sea $\operatorname{dim}(A)=d$ y supongamos que $A=C o(\operatorname{Ext}(A))$ sea válido para cualquier conjunto convexo y acotado tal 
que $\operatorname{dim}(A)<d$ (Hipótesis inductiva).

Sea $F$ una cara de $A$ tal que $F \neq A$. Como $\operatorname{dim}(F)<d$ y por la hipótesis inductiva $F=$ $C o(\operatorname{Ext}(F))$. Luego $\operatorname{Ext}(F) \subseteq \operatorname{Ext}(A)$ entonces $F \subseteq C o(\operatorname{Ext}(A))$. Por tanto $F \subseteq C o(\operatorname{Ext}(A))$, para toda cara $F$ de $A$ tal que $F \neq A$. Probemos ahora que $\operatorname{ri}(A) \subseteq C o(\operatorname{Ext}(A))$.

Sea $a \in \operatorname{ri}(A)$ dado que $r i(A)=r i(\bar{A})$, luego $a \in \operatorname{ri}(\bar{A})$ donde $\bar{A}$ es compacto. Por lema ??, existen $v_{1}, \ldots, v_{k} \in \operatorname{Ext}(\bar{A})$ con $(k \leq 2 d)$ tales que $a \in \operatorname{ri}\left(C o\left(\left\{v_{1}, \ldots, v_{k}\right\}\right)\right)$ y como $A$ es cara de $A$, por hipótesis $\operatorname{Ext}(\bar{A}) \subseteq \overline{\operatorname{Ext}(A)}$. Luego existen $a_{1}, \ldots, a_{k} \in \operatorname{Ext}(A)$ tales que $a_{j}$ esta suficientemente cerca de $v_{j}$ para $j \in\{1, \ldots, k\}$ y también $a \in \operatorname{ri}\left(C o\left(\left\{a_{1}, \ldots, a_{k}\right\}\right)\right)$. Por tanto $a \in C o(\operatorname{Ext}(A))$. Supongamos $A \neq C o(\operatorname{Ext}(A))$ luego existe $y \in A$ tal que $y \notin C o(\operatorname{Ext}(A))$ y por lo anterior $y \notin \operatorname{ri}(A)$ luego $y \in A \backslash \operatorname{ri}(A)=\operatorname{cl}(A)$ de donde existe un hiperplano de soporte $H$ de $A$ en $y$. Como $F=A \cap H$ es una cara de $A$ tal que $y \in F$, resulta $y \in F \subseteq \operatorname{Co}(\operatorname{Ext}(A))$, absurdo. Por lo tanto $A=C o(\operatorname{Ext}(A))$.

Recíprocamente, supongamos que $A=\operatorname{Co}(\operatorname{Ext}(A))$. Sea $F$ una cara de $A$, luego por definición de cara se tiene que:

$$
F=F \cap A=F \cap \operatorname{Co}(\operatorname{Ext}(A))=\operatorname{Co}(F \cap \operatorname{Ext}(A))=\operatorname{Co}(\operatorname{Ext}(F))
$$

por tanto $F=C o(E x t(F))$. Entonces basta probar el resultado para la cara $A$ de $A$, pues para el resto de caras se procederá de modo análogo. Por tanto supongamos que $\operatorname{Ext}(A)$ no es denso en $\operatorname{Ext}(\bar{A})$ y sin pérdida de generalidad supongamos $\operatorname{dim}(A)=n$, entonces existen un punto extremo $x$ de $\bar{A}$ y una vecindad abierta $V$ de $x$ en $\mathbb{R}^{n}$ tal que $V \cap \operatorname{Ext}(A)=\emptyset$. Sea $B=C o(\bar{A} \backslash V)$ convexo y compacto, pues $\bar{A} \backslash V$ es compacto. Además $x \notin B$, caso contrario como $x \in \operatorname{Ext}(\bar{A})$ entonces $x \in \bar{A} \backslash V$, absurdo. Luego existe un hiperplano $H$ que separa fuertemente a $x$ de $B$. Supongamos $x \in \operatorname{int} H^{+}$luego como $\operatorname{Ext}(A) \subseteq B$ entonces $A=\operatorname{Co}(\operatorname{Ext}(A)) \subseteq$ int $H^{-}$. De donde la vencidad de $x$ dada por $P=i n t H^{+} \cap V$ es tal que $P \cap A=\emptyset$, absurdo.

Teorema 3.3 Sea $A \subseteq \mathbb{R}^{n}$ no vacio, convexo y tal que no contiene líneas. Para cada cara $F$ de $A$ supongamos que $\operatorname{Ext}(F) \cup \operatorname{Rxt}(F)$ es denso en $\operatorname{Ext}(\bar{F}) \cup \operatorname{Rxt}(\bar{F})$. Entonces $A=\operatorname{Co}\left(\operatorname{Ext}(A) \cup \operatorname{Rxt}(A)^{\prime}\right)$.

Prueba. Usaremos inducción en la dimensión $d$ de $A$. Para $d \leq 1$ el resultado es trivial pues $A$ sería a lo más una semi recta. Sea $\operatorname{dim}(A)=d$ y supongamos que el teorema sea válido para cualquier conjunto convexo que no contiene líneas y tal que $\operatorname{dim}(A)<d$.

Sea $F$ una cara de $A$ tal que $F \neq A$. Entonces por hipótesis inductiva $F=\operatorname{Co}(\operatorname{Ext}(F) \cup R x t(F))$. Por otro lado, como $\operatorname{Ext}(F) \subseteq \operatorname{Ext}(A)$ y $\operatorname{Rxt}(F) \subseteq R x t(A)$ entonces $F \subseteq \operatorname{Co}(\operatorname{Ext}(A) \cup R x t(A))$, para toda cara $F$ tal que $F \neq A$. Veamos ahora que $\operatorname{ri}(A) \subseteq \operatorname{Co}(\operatorname{Ext}(A) \cup \operatorname{Rxt}(A))$. Sea $a \in \operatorname{ri}(A)$. Entonces existe un convexo acotado $C$ tal que $a \in C \subseteq \operatorname{ri}(A)$ luego aplicando el lema 3.1 al compacto $\bar{C}$, existen $v_{1}, \ldots, v_{k} \in \operatorname{Ext}(\bar{C})(k \leq 2 d)$ tales que $a \in \operatorname{ri}\left(\operatorname{Co}\left(\left\{v_{1}, \ldots, v_{k}\right\}\right)\right)$. Por otro lado $\bar{A}$ es cerrado, convexo y no contiene líneas luego admite representación extremal, luego por teorema $2.5 \bar{A}=\operatorname{Co}(\operatorname{Ext}(\bar{A}) \cup \operatorname{Rxt}(\bar{A}))$ de donde $v_{j} \in \operatorname{Ext}(\bar{C}) \subseteq \operatorname{Co}(\operatorname{Ext}(\bar{A}) \cup \operatorname{Rxt}(\bar{A}))$, luego para $j \in\{1, \ldots, k\}$ fijo existen $v_{1}^{j}, \ldots, v_{p(j)}^{j} \in \operatorname{Ext}(\bar{A})$ y $w_{1}^{j}, \ldots, w_{q(j)}^{j} \in \operatorname{Rxt}(\bar{A})$ que verifican $v_{j} \in C o\left(\left\{v_{1}^{j}, \ldots, v_{p(j)}^{j}, w_{1}^{j}, \ldots, w_{q(j)}^{j}\right\}\right)$ así entonces se tendrá:

$$
a \in \operatorname{ri}\left(\operatorname{Co}\left(\left\{v_{1}^{1}, \ldots, v_{p(1)}^{1}, w_{1}^{1}, \ldots, w_{q(1)}^{1}, \ldots, v_{1}^{k}, \ldots, v_{p(k)}^{k}, w_{1}^{k}, \ldots, w_{q(k)}^{k}\right\}\right)\right)
$$

también dado que $\operatorname{Ext}(A) \cup \operatorname{Rxt}(A)$ es denso en $\operatorname{Ext}(\bar{A}) \cup \operatorname{Rxt}(\bar{A})$ podemos suponer que $v_{1}^{j}, \ldots, v_{p(j)}^{j} \in \operatorname{Ext}(A)$ y $w_{1}^{j}, \ldots, w_{q(j)}^{j} \in \operatorname{Rxt}(A)$ basta aproximarnos lo suficiente, luego $a \in \operatorname{Co}(\operatorname{Ext}(A) \cup \operatorname{Rxt}(A))$.

Supongamos exista $y \in A$ tal que $y \notin C o(\operatorname{Ext}(A) \cup \operatorname{Rxt}(A))$, por lo anterior $y \notin \operatorname{ri}(A)$ luego $y \in \operatorname{cl}(A)$ de donde existe una cara $F$ de $A$ tal que $y \in F$, luego $y \in \operatorname{Co}(\operatorname{Ext}(A) \cup \operatorname{Rxt}(A))$, absurdo. Entonces $A=\operatorname{Co}(\operatorname{Ext}(A) \cup \operatorname{Rxt}(A))$. 
Nuevamente para mostrar el resultado de modo general necesitamos la noción de espacio de linealidad.

Teorema 3.4 Sea $A \subseteq \mathbb{R}^{n}$ no vacío y convexo. Si $L$ es el espacio de linealidad de $A$ y $A_{L}=A \cap L^{\perp}$. Para cada cara $F$ de $A_{L}$ supongamos que $\operatorname{Ext}(F) \cup \operatorname{Rxt}(F)$ es denso en $\operatorname{Ext}(\bar{F}) \cup \operatorname{Rxt}(\bar{F})$. Entonces

$$
A=L+\operatorname{Co}\left(\operatorname{Ext}\left(A_{L}\right) \cup \operatorname{Rxt}\left(A_{L}\right)\right)
$$

Prueba. Basta usar la relación $A=L+A \cap L^{\perp}$ y notar que $A_{L}=A \cap L^{\perp}$ satisface las hipótesis del teorema 3.3.

\section{Conclusiones}

4.1 Todo conjunto cerrado y convexo, admite representación extremal.

4.2 En todo conjunto cerrado y convexo que no admite rayos extremos la representación extremal(formada unicamente por puntos extremos) verifica la noción de minimalidad.

4.3 El trabajo caracteriza los conjuntos convexos y acotados para los cuales la tesis del teorema de Krein-Milman es válida.

4.4 Se muestra una condición necesaria para que un conjunto convexo arbitrario, admita representación extremal. 


\section{REFERENCIAS BIBLIOGRÁFICAS}

[1] Auslender, A. y Teboulle. Asymptotic cones and functions in optimization and variational inequalities. Springer-Velag, New York, 2003.

[2] Florenzano, M. Finite Dimensional Convexity and Optimization. Springer-Velag, Berlin, 2001.

[3] Hiriart-Urruty, J. B. L. Convex Analysis and Minimization Algorithms, SpringerVelag, Berlin-New York, 1993.

[4] Kelley, J. L. Note on a theorem of Krein and Milman. J. Osaka Inst. Sci. Technol. Part I, 3, 1951, pp. 1-2.

[5] Klee, V. L. Convex sets in linear spaces II. Duke math., 18, 1951, pp. 875-883.

[6] Klee V. L. Extremal structure of convex sets. Arch. Math., 8, 1957, pp. 234-240.

[7] Rockafellar. R. T. Convex Analysis. Princeton University Press, Princenton, NJ, 1970. 